\title{
Joint-action coordination in transferring objects
}

\author{
Ruud G. J. Meulenbroek • Jurjen Bosga • \\ Majken Hulstijn • Stephan Miedl
}

Received: 29 June 2006 / Accepted: 5 January 2007 / Published online: 26 January 2007

(C) Springer-Verlag 2007

\begin{abstract}
Here we report a study of joint-action coordination in transferring objects. Fourteen dyads were asked to repeatedly reposition a cylinder in a shared workspace without using dialogue. Variations in task constraints concerned the size of the two target regions in which the cylinder had to be (re)positioned and the size and weight of the transferred cylinder. Movements of the wrist, index finger and thumb of both actors were recorded by means of a 3D motiontracking system. Data analyses focused on the interpersonal transfer of lifting-height and movement-speed variations. Whereas the analyses of variance did not reveal any interpersonal transfer effects targeted data comparisons demonstrated that the actor who fetched the cylinder from where the other actor had put it was systematically less surprised by cylinder-weight changes than the actor who was first confronted with such changes. In addition, a moderate, accuracy-constraint independent adaptation to each other's movement speed was found. The current findings suggest that motor resonance plays only a moderate role in collaborative motor control and confirm the independency between sensorimotor and cognitive processing of action-related information.
\end{abstract}

Keywords Joint action - Size-weight illusion · Fitts' law · Grasping · Coordination

R. G. J. Meulenbroek $(\bowtie) \cdot J$. Bosga $\cdot$ M. Hulstijn $\cdot$ S. Miedl Nijmegen Institute for Cognition and Information, Radboud University Nijmegen, PO Box 9104, 6500 HE Nijmegen, The Netherlands e-mail: r.meulenbroek@nici.ru.nl

\section{Introduction}

Given the strong inclination that people have towards behavioral mimicry when they are jointly involved in walking or speaking (Chartrand and Bargh 1999; Lakin et al. 2003), can we also demonstrate co-actors adopting each other's movement patterns in the simple task of transferring objects? If an object relevant for my own actions is placed in front of me at a high speed, shall I then automatically pick it up and transfer it also with a high speed or will I stick to my own, preferred speed? Furthermore, will an observer be sensitive to the height of the movement trajectory with which an object is placed within reach as a potential cue of that object's weight?

Detailed questions surrounding the potential transfer of movement kinematics in a shared action sequence as specified above have not yet been systematically investigated (cf. Sebanz et al. 2006). The little work that has been done in this area was limited to unintentional synchronization of arm movements in pendulum swinging (Schmidt and O'Brien 1997), incidental entrainment of leg movements when dyads solve a puzzle through dialogue (Shockley et al. 2003), and the kinematic characteristics of cooperative versus competitive grasping movements (Georgiou et al. 2007).

A recent model of joint action by Oztop et al. (2005) that makes relevant claims regarding joint-action coordination in sequential task performance presumes that when a person observes someone else's actions she automatically will activate her own action system not only to understand the behavior of the actor being observed (Rizzolatti and Craighero 2004) but also to infer that actor's intentions (cf. Wolpert et al. 2003; Iacoboni et al. 2005). The example Oztop et al. (2005) 
mentioned in this context concerns the implied meaning that is conveyed by the specific way in which people may handle a tool. For example, whereas holding a hammer with a usual grip around its handle is very likely to signal the intent of hammering, holding the hammer with a power grip around its face communicates the intention to handing over the tool to someone else rather than start hammering with it oneself. Of course, which semantics are primed by the way in which a tool is handled will depend on the shared knowledge of how the tool is used for the general purpose it was designed for (see also Cuijpers et al. 2006).

In the present study we were neither interested in what a particular act might convey to an observer in terms of action semantics (Oztop et al. 2005) nor how cooperative or competitive contexts are differentially reflected in the prehension kinematics of dyads (Georgiou et al. 2007). Instead, we focused on the extent to which an observer in her subsequent movements is influenced by specific kinematic features of a co-actor's movements bringing the object within reach. Since the participants in the present study were asked to perform complementary acts rather than identical ones this research question is therefore only indirectly related to topics like behavioral mimicry and imitation (Koski et al. 2003).

Our reasoning about the possible transfer of kinematic movement parameters from one actor to another was that if during action observation people internally simulate the movements that they observe (Gallese and Goldman 1998; Rizzolatti and Craighero 2004), then the kinematic features of these simulated movements might as well be re-used for a related, self-generated movement that, in time, closely follows the observed one even when this future movement is complementary in nature. Economizing on parameter remapping costs formed the basis of this expectation (cf. Rosenbaum et al. 1986).

As regards inference making we were interested in the extent to which people derive the weight of an object when seeing someone else picking up and transporting the object. A similar matter has been investigated in an fMRI study by Grezes et al. (2004) but their experiment was primarily designed to find the brain correlates of action observation in the context of deception. To this end these researchers used pictures of an accomplice lifting boxes as if they were heavy or when they were actually heavy. In the present study, however, deceit was not the issue of investigation. Moreover, the object-weight dependent variations in movement kinematics were more subtle than in the Grezes et al. (2004) study and related studies on box lifting by Hamilton et al. (2007) and Kingma et al. (2005) as we will explain next.
To gain further insight into the extent to which taskdependent kinematic variations affect the subsequent complementary actions of a co-actor, we exploited two widely investigated motor-control paradigms, viz. the speed-accuracy trade-off (Fitts 1954; Fitts and Peterson 1964) and size-weight illusion (Charpentier 1891). Figure 1 depicts the transportation task that we used for this purpose. First, one of the two actors-the 'putting-actor'-was asked to pick up a vertically positioned cylinder from a nearby location on the table and to put that cylinder into a circular-shaped target area in the middle of the table, i.e., within reach for the other actor. Subsequently, the other actor-the 'fetchingactor'-was asked to fetch the cylinder from where the putting-actor had left it, and to reposition it, also in a circular-shaped, nearby target area. The fetching-actor was instructed not to pick up the cylinder before the putting-actor had released it.

Three task variables were manipulated, viz. the size of the target areas in which the actors were to successively reposition the cylinder and the size and weight of the to-be-transported cylinder. The size of the target areas was expected to modulate the actors' movementspeed and the size and weight properties of the cylinders were thought to affect the height with which the actors would lift the cylinders during transport. Before elaborating on our rationale it is important to mention that task conditions were kept constant within trial blocks of three repetitions to examine how quickly the dyads adapted to the between trial-block changes in the experimental conditions. All factors other than the

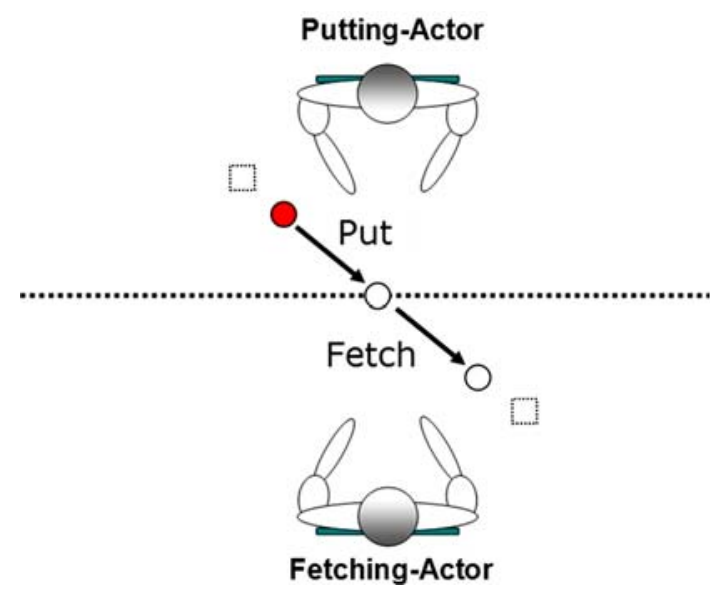

Fig. 1 Top view of experimental setup depicting the experimental task. The dashed squares represent the starting areas for the (right) hand movements of both actors. At the filled circle a cylinder was positioned which had to be picked up by the puttingactor, repositioned in the central circular target area where the fetching-actor was to pick it up and place it in the target circle in the vicinity of her own starting area 
repetition factor were randomized across the trial series and dyads, and at the start of each trial block the participants were unaware of the experimental conditions of that particular block (see "Method").

Two types of potential transfer of movement kinematics were foreseen in our experimental task. First, it was expected that an incorrectly anticipated weight of a to-be-picked up cylinder would be reflected in the height with which an actor would lift that cylinder (cf. Grezes et al. 2004). A lighter than expected cylinder was assumed to be lifted higher than a heavier one and vice versa, i.e., a heavier than expected cylinder was assumed to be lifted less high than a lighter one. Furthermore, if the fetching-actor would infer the weight of the cylinder that was transported by the putting-actor by observing her movements, then the fetching-actor was expected to have a more precise initial estimate of the weight of the to-be-transported cylinder than the putting-actor. The degree to which any surprise effect due to a wrongly anticipated object weight would transfer from the putting-actor to the fetching-actor thus formed a test as to whether or not the fetching-actor had actually inferred the cylinder weight. In repetitions two and three of each trial block both actors could rely, of course, on their sensorimotor memory (see Nowak and Hermsdorfer 2003) as regards the object weight since in these trials they already had handled that particular cylinder in the previous (two) trial(s) of the trial block.

To investigate the predicted object-weight dependent interpersonal kinematic transfer effects, we factorially combined two cylinder weights (light vs. heavy) with two cylinder sizes (small vs. large) yielding four cylinders. The size-weight illusion that results from this particular combination of object features reflects the fact that people perceive a smaller of two equally-massed objects as heavier. For one of the suggested explanations of this illusion, we refer to Flanagan and Beltzner (2000). The size-weight illusion is so prominent that it also yields its typical effects when wielding a small and a large object of equal weight in the left and right hand simultaneously suggesting the distorted perception is due to sensory invariants rather than inferences (Amazeen and Turvey 1996). Irrespective of any size-weight illusion effects, we expected the cylinder-weight variations to result in larger between trial adaptation effects on the height with which the participants would lift the cylinders than the cylinder-size variations (Gordon et al. 1991a, b). We thus examined the extent to which the weight of an object carried from one position to another would be inferred by an observer. If the observer, i.e., the fetching-actor, would not infer the weight of the transferred cylinder, then the surprise-effect expressed in the lifting height of the fetching-actor should be equally large as that in the putting-actor. Conversely, should the fetching-actor correctly infer the weight of the object by observing the putting-actor lifting and repositioning the cylinder, then the fetching-actor should be able to anticipate the cylinder's weight better and not show such large surprise effects as the putting-actor would.

A second manipulation targeted the extent to which the two actors would adopt each other's movement speed in the face of variations of movement-accuracy constraints. If merely by observing someone else's movements, one would be inclined to adopt a similar speed in a subsequent complementary movement, then confronting two actors with different target area sizes was expected to modulate their speed differently than when the actors would be confronted with these target areas individually. The rationale here was based on Fitts' law, which states that one's movement speed systematically varies with the size of the target area (Fitts 1954; Fitts and Peterson 1964; Mottet et al. 2001). The smaller the target area to reach for, the lower the movement speed due to the higher accuracy demands that are associated with small target areas. In the present study we manipulated the target area sizes of the first and the second actor separately, where the size was either small (diameter of $9 \mathrm{~cm}$ ) or large (diameter of $18 \mathrm{~cm}$ ). If both actors had identical (either small or large) target area sizes, their movement speeds were most likely to become similar under the assumption that there would be a strong tendency to adopt each others' speed. If this tendency of movement speed transfer was strong enough, then violations of Fitts' law in the fetching-actor could be expected under conditions in which the two actors were confronted with dissimilar target area sizes.

In sum, in the present study consisting of a sequential motor task involving two actors we tested whether lifting height - a parameter that potentially could reveal an object's weight—would be picked up by an observing co-actor and determine her subsequent handling of that object and whether she would be inclined to adapt her speed to the observed speed, or, alternatively, whether the interpersonal transfer of kinematic parameters would be incidental in nature, i.e., unrelated to the task constraints with which the individuals of the dyad were confronted.

\section{Method}

\section{Participants}

Fourteen right-handed adult dyads participated in the experiment. Their ages ranged from 18 to 30 years; mean age was $22 \pm 2$ years. The 28 participants, 22 
females and 6 males, formed 8 female dyads and 6 dyads of mixed gender. They received either course credits or payment (6 euros per hour) for their participation. Each participant signed an informed consent form. The study was approved by the local ethics committee and performed in accordance with the ethical standards laid down in the 1964 Declaration of Helsinki.

\section{Materials}

A Microsoft Windows-2000 controlled PC was used to control the stimulus events and data collection. Two synchronized 3D infrared motion-tracking systems (Optotrak $^{\mathrm{TM}}$, Northern Digital, Waterloo, Canada) were used to record the displacements of the wrist, thumb and index finger of the right hand of the two actors. Three infrared-light emitting diodes (IREDs) were used per participant. The IREDs were fixated on the wrist joint and the distal phalanxes of the index finger and thumb of the right arm of the participants. The IRED-position data were sampled at a rate of $100 \mathrm{~Hz}$ with accuracy better than $0.2 \mathrm{~mm}$ in $X, Y$ and $Z$ directions. Each participant wore a pair of earphones through which a repetitive sound was presented at approximately $60 \mathrm{~dB}$ throughout the experiment. This prevented the participants from speaking to each other and hearing any task-related noise, e.g., due to the cylinders making contact with the tabletop at completion of the transport phases. Each participant also wore a facial mask to avoid communication by means of facial cues. The participants were seated comfortably at opposite sides of a table on top of which a 1-cm thick marble plate was placed to prevent any object-weight information being conveyed from one actor to the other by means of vibrations via the table surface.

Four cylinders of $25 \mathrm{~cm}$ height were used, two with a diameter of $2.5 \mathrm{~cm}$ and two with a diameter of $6.5 \mathrm{~cm}$. Two cylinders had a lightweight of $230 \mathrm{~g}$ and two cylinders had a weight of $835 \mathrm{~g}$. To avoid any potential confounding between the effects of cylinder and targetarea size all cylinders were given a $4-\mathrm{mm}$ thick, circular base of $8 \mathrm{~cm}$ in diameter. This ensured that the thin and thick cylinders had the same size support base and could be repositioned in a comparable stable way which was a factor that also could contribute to the accuracy constraints since the participants were asked to place the cylinders on small and large circular target areas that were clearly marked on the table surface.

Procedure and task

The experiment consisted of three sections. In the first section one of the two participants performed 48 sin- gle-actor control trials consisting of picking up and repositioning in random order, but blocked (three repetitions per block), the four cylinders without the presence of the other participant. In the second section, the other participant performed 48 single-actor control trials, also alone. In the third section the two participants of the dyad performed 96 joint-action experimental trials. The 48 control trials per participant resulted from three blocked replications $(n=3)$ of each of the factorial combinations of the factors size of starting area (small vs. large), size of target area (small vs. large), cylinder weight (light vs. heavy) and cylinder size (small vs. large). The number of joint-action experimental trials was twice the number of single-actor control trials. In the joint-action experimental trials each participant was given in half the trials the role of putting the cylinder in the centre of the table, and in the other half of the trials the role of fetching the cylinder from the centre of the table (see Fig. 1). The role was randomly assigned to the trial blocks of three repetitions.

In their control trials the participants of dyads 1-9 (arbitrary numbered) performed the fetch-part of the experimental task, i.e., at the start of their control trials they were confronted with a cylinder that was placed in the middle of the table and their task was to pick it up and place it in the area which was closest to their own starting position. Dyads 10-14 performed in their control trials the put-part of the experimental task, i.e., at the start of each trial they found the cylinder on the area closest to themselves and they were instructed to put the cylinder in the target area at the centre of the table. The data analyses showed that there were no systematic differences between the two groups of dyads as far as the targeted research questions concerned so the comparisons with the control trial data will not be addressed further in the remainder of this paper.

The experiment was conducted by two experimenters who sat behind the participants. Each experimenter had a clear view on a separate computer screen which displayed information that specified the experimental conditions of every trial. In-between trials the participants were asked to close their eyes. After both participants had closed their eyes, the experimenters put the appropriate target pads on the table as well as the appropriate cylinder in the starting area of the puttingactor. Then, the two experimenters simultaneously tapped the participants on their lower arm, which indicated that they could open their eyes to start performing the trial. At the same time one of the experimenters started the movement recording.

The standard event sequence in a joint-action experimental trial was as follows. At the start of each trial 
one of the two actors, i.e., the putting-actor, found in a location close to the starting position of the right hand a cylinder that had to be picked up and moved to the target area in the centre of the tabletop (see Fig. 1). Only after the putting-actor had released the cylinder was the fetching-actor allowed to pick up the cylinder and to reposition it in a target area adjacent to her. The action sequence was to be completed within $8 \mathrm{~s}$ during which the displacements of the wrist and finger IREDs on both actors were sampled at a rate of $100 \mathrm{~Hz}$. After every 48 trials, or whenever they asked, the participants were offered a 10-min break.

\section{Data analysis}

The raw IRED position data were smoothed with a second-order, low-pass Butterworth filter with a cut-off frequency of $8 \mathrm{~Hz}$. Subsequently, the position data were segmented on the basis of the tangential velocitytime functions of the IREDs on the wrists of the two actors (see top-left graph in Fig. 2).

The movement sequence of each actor was segmented into three intervals, the first interval corresponding to grasping the cylinder starting with the right hand in the home-position on the edge of the table and ending with finger and thumb making contact with the object, the second interval corresponding to the transportation of the cylinder to the target area (see put and fetch legends in Fig. 2), and the third interval corresponding to the participant's hand returning to the home-position. The intervals were detected by a semiautomatic search procedure for relevant local minima in the tangential velocity profiles of the wrist movements. The time indexes thus found could be adjusted with an interactive computer program using a crosshair whenever the experimenter considered the segmentation inaccurate.

Following segmentation, the data analyses focused on the displacements of the IREDs located on the tip of the thumb and index finger of each of the two actors. The top-right graph of Fig. 2 shows a 3D-graph of the typical finger-thumb displacements of the puttingactor (in green or light grey) and the fetching-actor (in red or dark grey). The three cylinders were added to this graph post hoc to indicate the phasing of the displayed movement patterns. The bottom-left graph of Fig. 2 shows the aperture-time functions of both actors. This figure was used to inspect the grasping behavior of the participants. Systematic aperture overshoots just prior to object contact and upon object release were visually checked by the experimenter to verify whether the task of each actor was performed as requested.

The bottom-right graph of Fig. 2 shows the height in the $Z$-dimension (perpendicular to the table surface) of the midpoint between the 3D positions of the IREDs on the thumb and index finger of each actor. This parameter reflected the surprise effects due to unexpected between-trial cylinder-weight changes on the lifting height, which was defined as the difference in the vertical position of the midpoint between the tips of the digits at the time of grasping the cylinder and the maximum height realized during transportation. In the second interval of each of the three-segment movement patterns, i.e., the green function for the
Fig. 2 Kinematics derived from position data of infraredlight emitting diode (IRED) on tip of the thumb, tip of the index finger and wrist as obtained in a prototypical trial. Green (light grey) functions are from the putting-actor, red (dark grey) functions are from the fetching-actor. The topleft graph shows the tangential wrist-velocity time functions, the top-right graph a 3D rendering of finger-thumb displacements, the bottom-left graph shows the aperturetime functions, and the bottom-right graph shows the height (in $\mathrm{cm}$ above the table surface) of the midpoint between the thumb and indexfinger IREDs
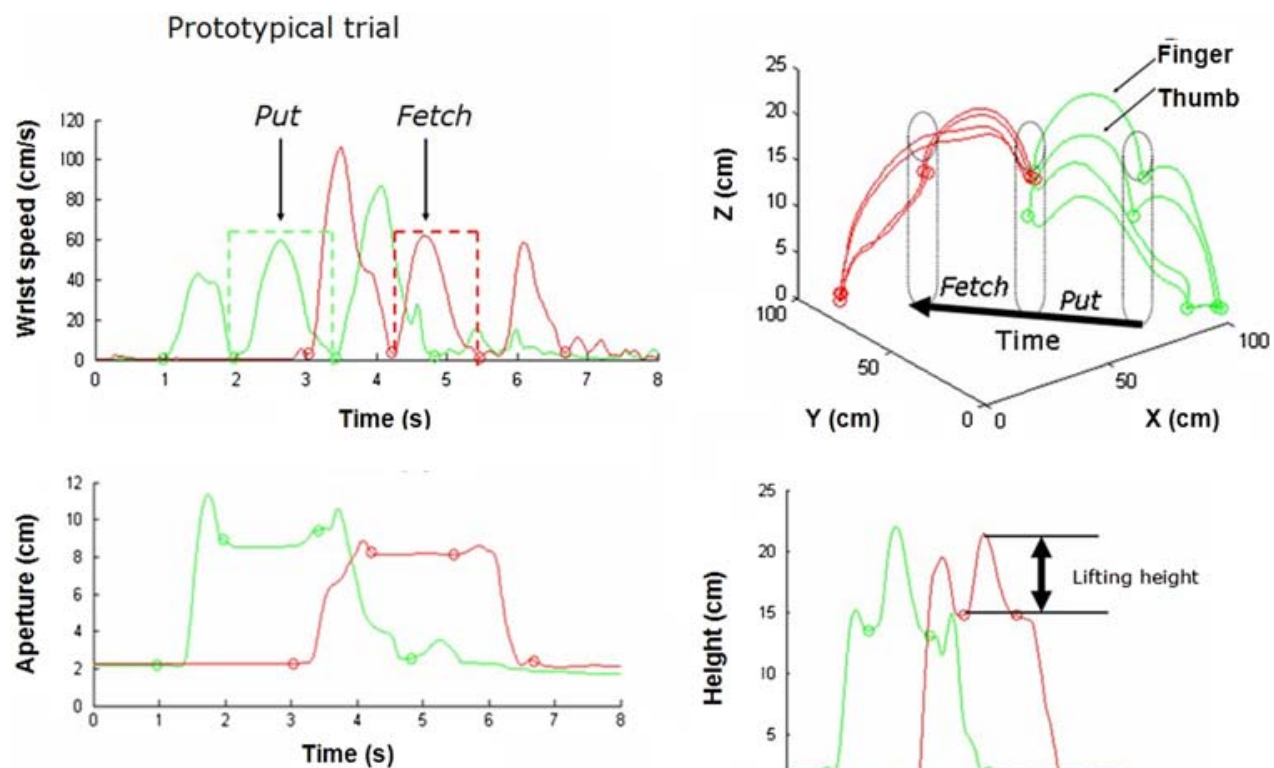
putting-actor and the red function for the fetchingactor, this lifting height reflected the height with which the participants lifted the cylinder while transporting it to their target area.

The maximum lifting height (in $\mathrm{cm}$ ) and the mean tangential wrist speed (in $\mathrm{cm} / \mathrm{s}$ ) realized by each actor during cylinder transport were used for further analyses. Statistical evaluation of the data consisted of repeated measures ANOVAs of which the designs are described in the relevant paragraphs in the results section or targeted $t$ tests to evaluate specific hypotheses in more detail.

\section{Results}

\section{Effects of cylinder properties}

Figure 3 shows the means and standard errors of the lifting heights realized by the putter and fetcher as a function of cylinder mass, cylinder size and the repetition factor. Table 1 summarizes the results of the repeated measures ANOVA that was conducted to evaluate these effects with a factorial design consisting of the within-dyad factors 2 roles (put vs. fetch) $\times 2$ object masses (light vs. heavy) $\times 2$ object sizes (small vs. large diameter $) \times 3$ repetitions. As expected, the light cylinders were lifted higher than the heavy ones $[F(1,13)=86.5, P<0.001]$. This effect interacted with object size $[F(1,13)=14.4, P<0.01]$ in line with the size-weight illusion. For the light cylinders it was the smaller one that was lifted higher than the larger one. For the two heavy cylinders, it was the larger one that was lifted higher than the smaller one. The interaction between object mass and object size on lifting heightreflecting the size-weight illusion effect-was most pronounced in repetition 1 as confirmed by the firstorder interaction between object mass and repetition $[F(2,26)=123.8, P<0.001]$ but remained present in repetitions 2 and 3 as reflected by the second-order interaction between object mass, object size and repeti- tion $[F(2,26)=3.78, P<0.05]$, confirming the robustness of the size-weight illusion.

No main effect or interaction of role (put vs. fetch) was found (all Fs $<1$ ), which was not unexpected since cylinder-weight increases and decreases were expected to have counteracting effects on the lifting heights by the putter and fetcher if any interpersonal transfer would take place at all. Targeted paired $t$ tests confirmed that there were systematic interpersonal transfer effects of lifting height between putter and fetcher as will be described next.

A detailed time-series analysis of the lifting heights which the dyads realized indicated that the fetchingactor benefited from movement observation. The results of this analysis are shown in Fig. 4. In those cases in which from one trial block to the next the cylinder's weight changed from heavy to light and the putting-actor showed a 'surprise' effect as reflected by an increase of her lifting height between these two trials of, on average, $4.69 \mathrm{~cm}$, the fetching-actor showed a similar surprise effect but to a smaller extent. This reduced surprise effect in the fetching-actor of, on average, $3.67 \mathrm{~cm}$, is shown in the left-hand graph of Fig. 4. The incidence of this effect was relatively high. It happened in $88 \%$ of those trials (i.e., $N=99$ ) in which a heavy-to-light cylinder-weight change occurred. A two-tailed $t$ test comparing the lifting heights on trial $i-1$ and trial $i$ (being the third trial of each trial block and the first of the subsequent block, respectively) for the putting-actor revealed that her surprise effect was statistically significant $[t(98)=15.98, P<0.01]$. For the fetching-actor, the smaller surprise-effect was also statistically significant $[t(98)=13.54, \quad P<0.01]$. A repeated measures ANOVA confirmed that the relevant interaction between role (putter vs. fetcher) and trial $(i-1=$ heavy and $i=$ light $)$ was statistically significant $[F(1,13)=7.24, P<0.05]$.

We found a similar effect when focusing on those trial pairs in which the cylinder-weight changed from light to heavy and the putter showed a 'surprise' effect
Fig. 3 Means and standard errors of the lifting height (in $\mathrm{cm}$ ) as a function of role (putting vs. fetching), object diameter (small vs. large) and object weight (light vs. heavy) for repetitions 1,2 and 3 separately

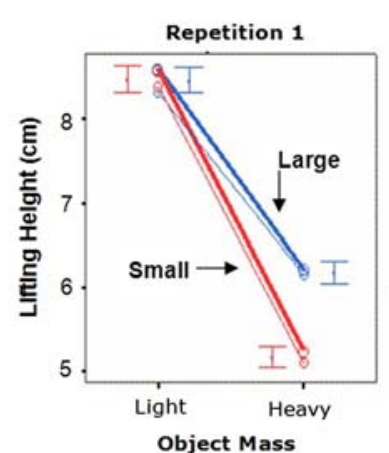

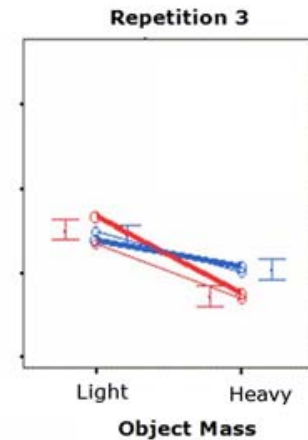

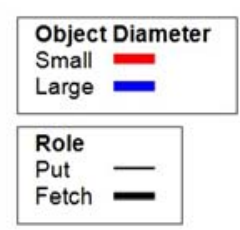

Object Mass

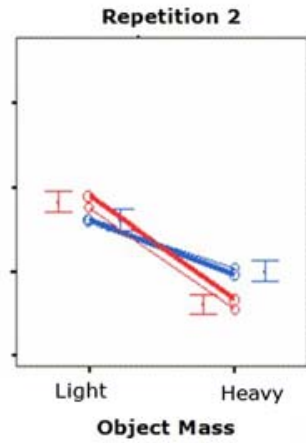


Table 1 Results of repeated measures Anova on the lifting height according to a 2 role (putting vs. fetching) $\times 2$ object mass $(230$ vs. $835 \mathrm{~g}) \times 2$ object sizes $(2.5$ vs. $6.5 \mathrm{~cm}$ in diameter $) \times 3$ repetitions factorial design

\begin{tabular}{llll}
\hline Factor & $d f$ & $F$ & $P$ \\
\hline Role & 1,13 & $<1$ & NS \\
Object mass & 1,13 & 86.5 & $<0.001$ \\
Object size & 1,13 & 7.1 & $<0.05$ \\
Repetition & 2,26 & 58.0 & $<0.001$ \\
$\begin{array}{l}\text { Role } \times \text { object mass } \\
\text { Role } \times \text { object size }\end{array}$ & 1,13 & $<1$ & $\mathrm{NS}$ \\
$\begin{array}{l}\text { Object mass } \times \\
\text { object size }\end{array}$ & 1,13 & 1.3 & $\mathrm{NS}$ \\
$\begin{array}{l}\text { Role } \times \text { object mass } \times \\
\text { object size }\end{array}$ & 1,13 & 14.4 & $<0.01$ \\
$\begin{array}{l}\text { Role } \times \text { repetition } \\
\text { Object mass } \times \\
\text { repetition }\end{array}$ & 1,13 & $<1$ & $\mathrm{NS}$ \\
$\begin{array}{l}\text { Role } \times \text { object mass } \times \\
\text { repetition }\end{array}$ & 2,26 & $<1$ & $\mathrm{NS}$ \\
$\begin{array}{l}\text { Object size } \times \text { repetition } \\
\text { Role } \times \text { object size } \times\end{array}$ & 2,26 & 123.8 & $<0.001$ \\
$\quad$ repetition \\
$\begin{array}{l}\text { Object mass } \times \text { object size } \times \\
\text { repetition }\end{array}$ & 2,26 & $<1$ & $\mathrm{NS}$ \\
$\begin{array}{l}\text { Role } \times \text { object mass } \times \\
\text { object size } \times \text { repetition }\end{array}$ & 2,26 & 3.67 & $<0.05$ \\
\hline & 2,26 & $<1$ & $\mathrm{NS}$ \\
\hline
\end{tabular}

as reflected by a decrease of her lifting height between those trials. The results of this analysis are shown in the right-hand graph of Fig. 4. Whereas the putting-actor proved to lift the cylinder, on average, $2.21 \mathrm{~cm}$ less high when the cylinder turned heavier from trial $i-1$ to trial $i$, the fetching-actor did so as well but, on average, for only $0.97 \mathrm{~cm}$. The incidence of this phenomenon was at chance level, i.e., in $54 \%(N=61)$ of the cases in which such a cylinder-weight change occurred in the experiment. A two-tailed $t$ test comparing the lifting

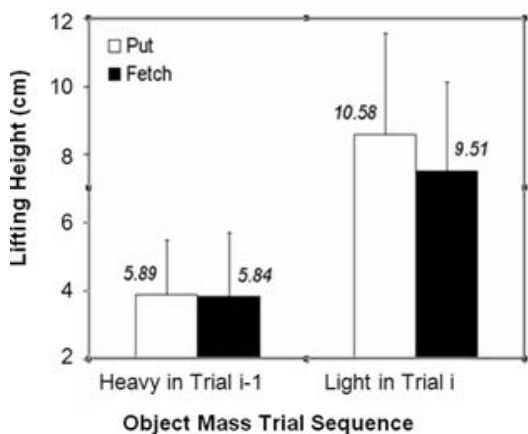

Fig. 4 Means and standard errors of the lifting height (in $\mathrm{cm}$ ) of the putting-actor and the fetching-actor. Left-hand graph: whenever the putting-actor showed a lifting-height increase from trial $i-1$ to trial $i$ due to an unexpected mass reduction between these trials, the fetching-actor also showed this surprise effect but less strong. Trials $i-1$ were the third trials of the trial blocks; trials $i$ heights on trial $i-1$ and trial $i$ for the putting-actor and fetching-actor separately again revealed that the effects were statistically significant for both actors $[t(60)=12.08, P<0.01, t(60)=3.69, P<0.01$, respectively]. A repeated measures ANOVA confirmed that the relevant interaction between role (putter vs. fetcher) and trial ( $i-1=$ light and $i=$ heavy) was statistically significant $[F(1,13)=31.08, P<0.001]$.

The time-series analyses on light-to-heavy cylinder weight changes and vice versa were corroborated by the results of dyad-based linear regression analyses in which the lifting height of the putting-actor in the first trial of the trial blocks was used as predictor for that of the fetching-actor. In contrast to the repeated measures ANOVA, the regression analysis took into account lifting-height co-variations in both directions, i.e., increases as well as decreases. An example of these data is depicted in Fig. 5. The slopes of the resulting regression equations turned out to be smaller than 1.0 for all 14 dyads showing that the putting-actor was more surprised by a cylinder-weight change than the fetching-actor. For nine of the dyads the positive slope proved statistically significant $(P<0.05)$. On average, the slopes amounted to 0.43 $\left(R^{2}=0.25\right)$, indicating that the fetching-actors were less surprised by any cylinder-weight change than the putting-actors.

\section{Effects of target-area size variations}

Figure 6 shows the average effects on the mean tangential wrist velocity of the putting and fetching-actors as a function of the manipulations of the size of the target regions where the cylinders had to be placed. The second part of the sequential action under study, i.e., fetch-

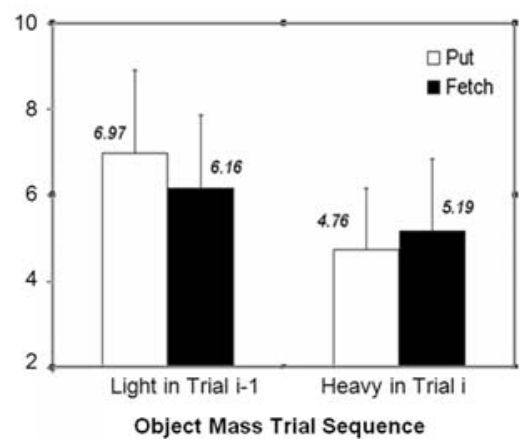

were the first trials of the subsequent trial blocks. Right-hand graph: similarly, whenever the putting-actor showed a liftingheight decrease from trial $i-1$ to trial $i$ due to an unexpected mass increase between these trials, the fetching-actor also showed this surprise effect, but again, not as strong as the putting-actor did 


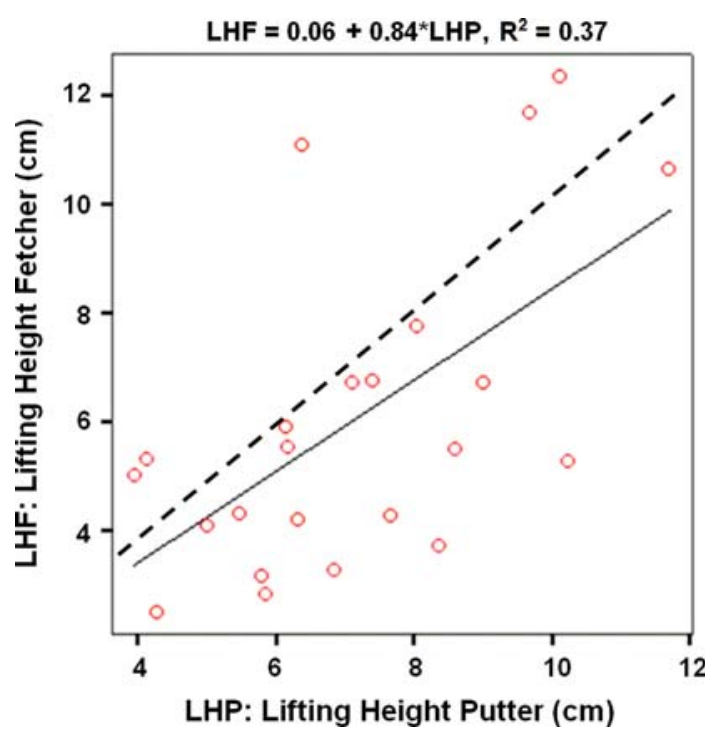

Fig. 5 Example of the linear regression analysis between the lifting-height data generated by the putter and fetcher of 1 of the 14 dyads participating in the study. The data concern the first trial within trial blocks of three repetitions of the experimental conditions. The regression analyses revealed the robustness of the observed reduction of the size-weight illusion due to movement observation in the dyads studied. The dashed line with slope $=1$ represents the situation in which the lifting height of the fetcher would equal that of the putter

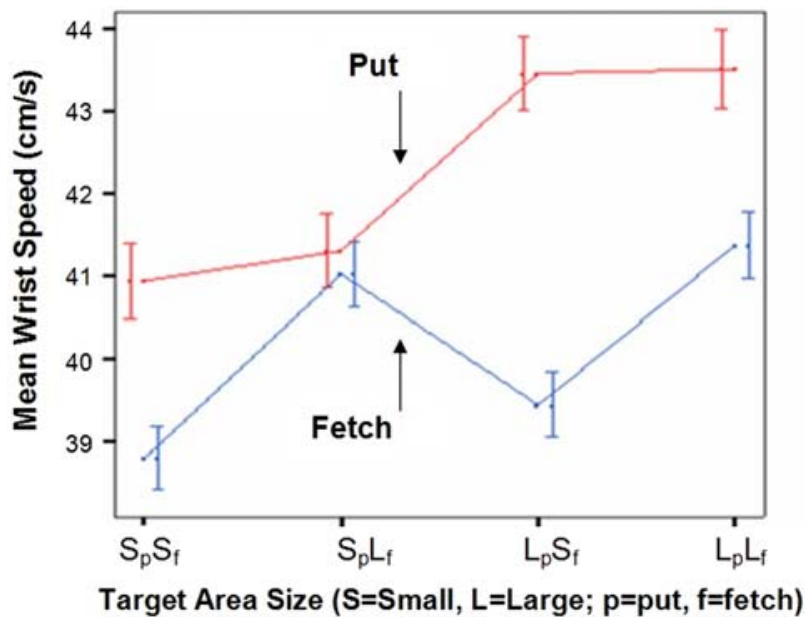

Fig. 6 Means and standard errors of the mean wrist speed (in $\mathrm{cm} / \mathrm{s}$ ) as a function of role (putting vs. fetching) and target area size ( $S=$ Small, $L=$ Large; $p=$ put, $f=$ fetch). The labels with subscript $p$ reflect the size of the target area of the putting-actor and labels with subscript $f$ reflect the size of the target area of the fetching-actor

ing the cylinder from the middle of the table, proved for no apparent reason overall to be performed somewhat slower than the first part, i.e., putting the cylinder in the middle of the table. The performance speeds of the participants were independently tuned to the sizes of the target areas with which the actors were confronted. In line with Fitts' law, small target areas elicited low speeds and large ones yielded high speeds. This relationship applied to both actors whether or not they were confronted with similar or dissimilar accuracy constraints. Table 2 summarizes the repeated measures ANOVA to evaluate these effects with a 2 roles (put vs. fetch) $\times 2$ sizes of putting target region ('putting-size'; small vs. large) $\times 2$ sizes of fetching target region ('fetching-size'; small vs. large) factorial design. The absence of the first-order interaction between puttingsize and fetching-size $(F<1)$ and the second-order interaction between the factors role, putting-size and fetching-size confirm the robustness of these findings.

A trial-by-trial inspection of the speed changes of both actors revealed, however, a moderate degree of performance-speed adaptation by the fetching-actor to that of the putting-actor. Figure 7 shows the results of this analysis. If from one trial to the next the size of the target areas remained constant for both actors but the putting-actor increased her performance speed for some reason, the fetching-actor followed this incidental speed increase of the putting-actor only partially as shown in the left-hand graph of Fig. 7. The incidence of this covariation in performance speed between the two actors was below chance level, i.e., it happened in $34 \%$ $(N=150)$ of the cases in which the phenomenon could theoretically occur. Nevertheless, the changes in speed were, in both actors, statistically significant $[t(149=7.63$, $P<0.02$ and $t(149)=5.95, P<0.01$, two-tailed, for the putting-actor and fetching-actor, respectively).

The reverse co-variation of performance speeds happened slightly more often. The right-hand graph of Fig. 7 shows that whenever the putting-actor reduced her movement speed from trial $i-1$ to trial $i$ during which the size of the target regions remained constant, the fetching-actor again partially followed this speed decrease. This co-variation in speed occurred in $42 \%$ $(N=186)$ of the cases in which this effect could occur. For the putting-actor and fetching-actor the speed

Table 2 Results of repeated measures ANOVA on the mean wrist speed (in $\mathrm{cm} / \mathrm{s}$ ) according to a 2 role (putting vs. fetching) $\times 2$ putting-size (small vs. large target area) $\times 2$ fetching-size (small vs. large target area) factorial design

\begin{tabular}{llll}
\hline Factor & $d f$ & $F$ & $P$ \\
\hline Role & 1,13 & 18.60 & $<0.01$ \\
Putting-size & 1,13 & 75.07 & $<0.001$ \\
Fetching-size & 1,13 & 33.90 & $<0.001$ \\
Role $\times$ putting-size & 1,13 & 20.21 & $<0.001$ \\
Role $\times$ fetching-size & 1,13 & 22.03 & $<0.001$ \\
Putting-size $\times$ fetching-size & 1,13 & $<1$ & NS \\
Role $\times$ putting-size $\times$ fetching-size & 1,13 & $<1$ & NS \\
\hline
\end{tabular}



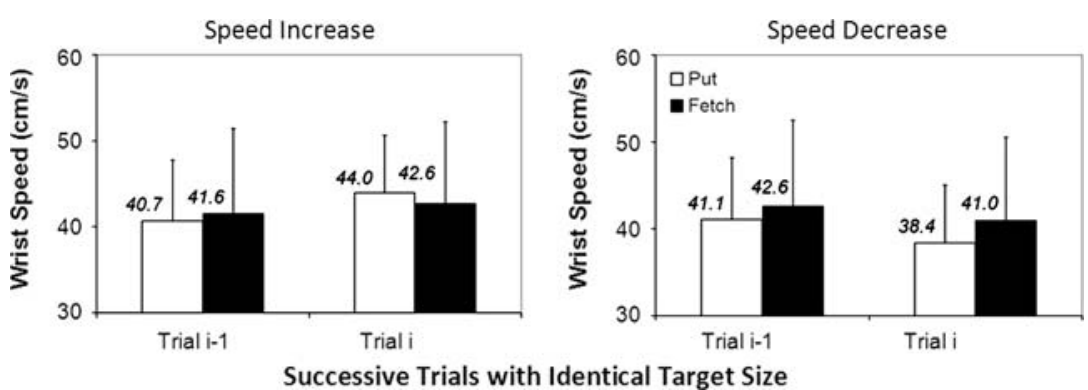

Fig. 7 Means and standard errors of the wrist speed (in $\mathrm{cm} / \mathrm{s}$ ) of the putting-actor and the fetching-actor. Left-hand graph: whenever the putting-actor showed a task-unrelated speed increase from trial $i-1$ to trial $i$, the fetching-actor also showed such a speed increase. The trials involved concerned the second and

decreases that were isolated here were again both statistically significant $[t(185)=5.95, \quad P<0.01$ and $t(185)=3.32, P<0.01$, two-tailed, respectively].

Linear regression analyses in which the mean wrist speeds of the putting-actor as observed in the second and third trials of the trial blocks were used as predictor for that of the fetching-actor confirmed the just described results. The slopes of the regression equations proved positive for 12 of the 14 dyads indicating that the fetchingactor followed the speed changes of the putting-actor in direction but only partially in size. For 10 of the 14 dyads the positive correlations proved statistically significant $(P<.05) . R^{2} \mathrm{~s}$ in these analyses ranged between 0 and 0.33 (mean $R^{2}$ was 0.11 ) indicating the target-size independent speed relationship between the two actors was weak.

Combined effects of size-weight illusion

and speed-accuracy trade-off

An inspection of the combined effects of the three task constraints that were manipulated revealed that even though on average the heavier cylinders were transported at a systematically lower speed than the lighter cylinders (mean $=43.78 \mathrm{~cm} / \mathrm{s} ; \mathrm{SD}=8.37 \mathrm{~cm} / \mathrm{s}$ for the light cylinders and $M=38.14 \mathrm{~cm} / \mathrm{s} ; \mathrm{SD}=7.06 \mathrm{~cm} / \mathrm{s}$ for the heavy cylinders), the speed variations due to the variations in the size and weight of the cylinders did not differentially affect the movement-speed variations as a result of the target-area size variations. Lifting-height variations due to the spatial accuracy-constraint manipulations were marginal $(<2 \mathrm{~mm})$ and also did not systematically influence the lifting-height variations due to the size-illusion effect.

\section{Discussion}

The present study examined the extent to which at the level of movement kinematics a transfer of performance third trials of the trial blocks in which no task conditions changed. Right-hand graph: similarly, whenever the putting-actor showed a task-unrelated speed decrease from trial $i-1$ to trial $i$, the fetching-actor followed this speed decrease

parameters takes place between co-actors involved in transferring objects. The results of the main analyses that we conducted to assess the effect sizes of the experimental manipulations were largely negative. Object-mass variations induced lifting-height variations of equal size in both actors. Target-region size based speed variations also did not show interpersonal transfer. These results suggest that in sequential joint actions the tuning to each others movement parameters is absent and thus demonstrate the limits of automatic movement simulation by observation as suggested by mirror-neuron theories (Gallese and Goldman 1998; Gallese et al. 2004; Rizzolatti and Craighero 2004). However, it remains to be seen whether the mirror-neuron system represents observed actions with a precision that allows for direct imitation. This does not appear to be the case in macaque monkeys. One could claim that any hypothetical system in general could infer the goals/intentions of observed movements without explicitly representing, say, the speed of the movement.

More detailed analyses of incidental interpersonal transfer effects indicated that the situation was more complex than that. When focusing on the effects of between-trial cylinder-weight changes, the actor who was confronted with such changes first showed a systematically larger surprise-effect than the actor who was asked to transport the cylinder after the first actor had done so. A key finding here was that through the realized trajectory height during object transportation, the object mass was picked up by movement observation and integrated into the observer's movement plan. Our prediction was therefore confirmed, i.e., the movements of an actor are likely to affect a subsequent, complementary movement generated by an observer.

Regarding the size of the effects it proved that the object property mass was, via lifting height, a more prominent factor determining task performance of the 
co-actors than movement speed. The linear regression analyses of the between-trial lifting-height changes due to cylinder-mass changes yielded larger proportions of explained variance when correlating the behavior of the fetching-actor with that of the putting-actor than the comparable analyses involving the incidental speed changes. With respect to goals and means of task performance, the dominance of object-mass over targetwidth makes sense given that the object is more likely to function as a goal in the task of handing over objects than the movements needed to perform such a task (Cuijpers et al. 2006). In a similar vein, the different sizes of the effects of object mass and movement speed could be taken to provide support for the importance of goal-inference making in collaborative joint action over and above a quasi-automatic, direct-matching based process of movement imitation or 'motor resonance' (Flanagan and Johansson 2003; Iacoboni et al. 1999; Koski et al. 2003) (Fig. 8).

Our interpretation of the current results does rely, of course, on the validity of both the weight-change effects and the co-variation of the actors' movement speeds. It could be argued that the fetcher's movement speed covaried with that of the putter due to a non-specific, visually primed increase in attention rather than the direct consequence of perceiving the putter's

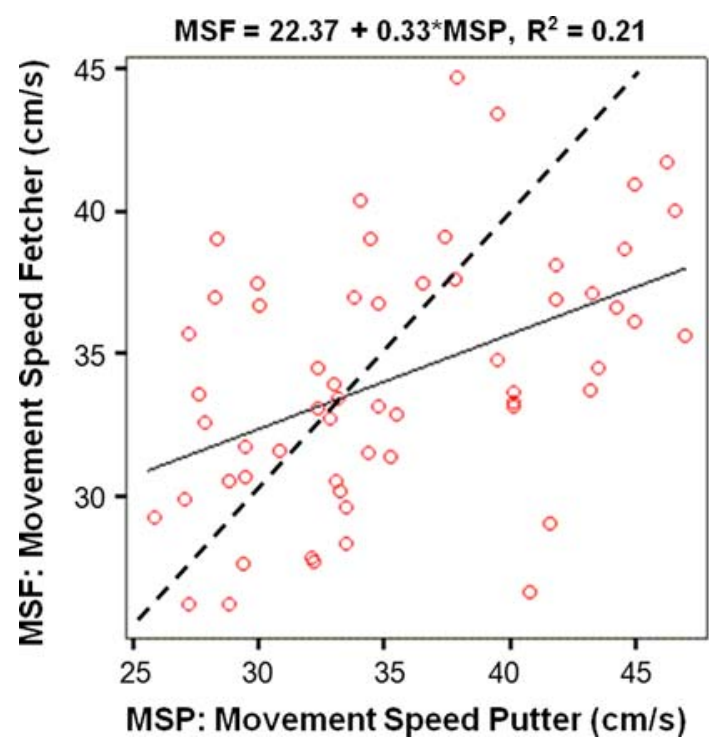

Fig. 8 Example of the linear regression analysis between the wrist-speed data generated by the putter and fetcher of 1 of the 14 dyads participating in the study. The data concern the second and third trials within trial blocks of three repetitions of the experimental conditions. The regression analyzes revealed the robustness of the observed, task-constraint independent covariation of movement speed between the two actors. The dashed line with slope $=1$ represents the situation in which the wrist speed of the fetcher would equal that of the putter object-transportation movements. However, attentional mechanisms are an unlikely explanation for our observations since the only visual cue that could have increased the attention in the fetcher was the putter's movement speed. Whether the object-weight related adaptation in the fetcher to the observed movement of the putter was due to the inference of the weight of the object or to the observed kinematics per se, also remains a matter of discussion. If it was the former, then motor resonance (i.e., mirror-neuron system) may not be responsible for a direct transfer of the kinematics parameters.

The effects of the object and the accuracy constraints differ in one fundamental aspect. The expected information transfer for the object is valid for the programming of the movement, while any hypothesized transfer of speed, by simple copying the observed movement to reduce dimensionality violates Fitts' law. This implies that our hypothesis about the transfer of speed was either not valid or that the effect is intrinsically short lived because the duration of the effect is overruled by intrapersonal constraints. In other words, while the information about weight change is very relevant for movement programming, the examined speed changes were not.

Apparently, movements that are being observed in a joint-action task can be used for multiple purposes. First, the observation might lead to internal simulation in order to understand the behavior that is being observed (Rizzolatti and Craighero 2004). Second, the internally simulated movements might provide clues as to the intentions associated with the observed behavior (Oztop et al. 2005; Wolpert et al. 2003; Iacoboni et al. 2005). Third, the observed movements might form the basis for prediction as to what an actor might do in the near future (Csibra 2005). Fourth, observed movements might provide clues about the task constraints at hand. In the present experiment, lifting height was such a parameter that indicated to the observer the weight of the cylinder that was transported. Fifth, observed movements may provide a global scaling parameter of ones own future actions, specifically if these actions are complementary to those performed by the actor that is being observed. Further research into the factors that determine the relative importance of these various roles which action observation may play in joint action is clearly needed.

Our study also suggests that kinematic parameters picked up during movement observation may even affect a subsequent movement that is performed in a workspace that is totally differently oriented than the workspace of the actor being observed. This finding extends the findings of the recent study by Van Schie 
et al. (2004) who showed that action observation activates cortical motor areas as if the observer had performed the task herself, i.e., when an observer who is facing an actor sees that actor move with her right hand the right cortical motor areas of the observer become activated indicating that the observer would have executed the task with her left hand. These findings suggest that movement simulation is egocentric and viewpoint-dependent.

Our demonstration of interpersonal action coordination in complementary joint action was restricted to the successive transportation of a cylinder by two actors. The movements preceding and succeeding the actual transportation of the cylinder could also have been scrutinized for signs of action coordination. However, the amplitudes of these movements by the two actors were hardly comparable. Moreover, the dyads involved in this study showed, not surprisingly, also the tendency to wanting to start task performance simultaneously. The preference of people to adopt either in-phase or out-of-phase timing patterns is not only prevalent in individual, inter-limb coordination tasks but also when people perform tasks together (Schmidt and O'Brien 1997; Shockley et al. 2003). These tendencies clearly coexist alongside the casual transfer of kinematic movement parameters in dyadic action sequences.

Acknowledgments The present study was supported by the EU-Project "Joint Action Science and Technology" (IST-FP6003747).

\section{References}

Amazeen EL, Turvey MT (1996) Weight perception and the haptic size-weight illusion are functions of the inertia tensor. J Exp Psychol Hum Percept Perform 22:213-232

Charpentier A (1891) Analyse experimentale de quelgues elements de la sensation de poids. Arch Physiol Norm Pathol 3:122-135

Chartrand TL, Bargh J (1999) The chameleon effect: the perception-behavior link and social interaction. J Pers Soc Psychol 76:893-910

Csibra G (2005) Mirror neurons and action observation. Is simulation involved? Publication of the European Science Foundation, retrieved 2 May 2006 from http://www. interdisciplines.org/mirror

Cuijpers RH, van Schie HT, Koppen M, Erlhagen W, Bekkering $\mathrm{H}$ (2006) Goal and means in action observation: a computational approach. Neural Netw 19:311-322

Fitts PM (1954) The information capacity of the human motor system in controlling the amplitude of movement. J Exp Psychol 47:381-391

Fitts PM, Peterson JR (1964) Information capacity of discrete motor responses. J Exp Psychol 67:103-112

Flanagan JR, Beltzner MA (2000) Independence of perceptual and sensorimotor predictions in the size-weight illusion. Nat Neurosci 3:737-741
Flanagan JR, Johansson RS (2003) Action plans used in action observation. Nature 424:769-771

Gallese V, Goldman A (1998) Mirror neurons and the simulation theory of mind-reading. Trends Cogn Sci 2:493-501

Gallese V, Keysers C, Rizzolatti G (2004) A unifying view of the basis of social cognition. Trends Cogn Sci 8:396-403

Georgiou I, Becchio C, Glover S, Castiello U (2007) Different action patterns for cooperative and competitive behaviour. Cognition. doi:10.1016/j.cognition.2006.01.008 (in press)

Gordon AM, Forssberg H, Johansson RS, Westling G (1991a) Visual size cues in the programming of manipulative forces during precision grip. Exp Brain Res 83:477-482

Gordon AM, Forssberg H, Johansson RS, Westling G (1991b) The integration of haptically acquired size information in the programming of the precision grip. Exp Brain Res 83:483488

Grezes J, Frith CD, Passingham RE (2004) Inferring false beliefs from the actions of oneself and others: an fMRI study. Neuroimage 21:744-750

de C Hamilton AF, Joyce DW, Flanagan JR, Frith CD, Wolpert DM (2007) Kinematic cues in perceptual weight judgement and their origins in box lifting. Psychol Res (in press)

Iacoboni M, Woods RP, Brass M, Bekkering H, Mazziotta JC, Rizzolatti G (1999) Cortical mechanisms of human imitation. Science 286:2526-2528

Iacoboni M, Molnar-Szakacs I, Gallese V, Buccino G, Mazziotta JC, Rizzolatti G (2005) Grasping the intentions of others with one's own mirror neuron system. PLoS Biol 3(e79):529-535

Kingma I, Van Dieën JH, Toussaint (2005) Scaling of lifting forces in relation to object size in whole body lifting. Ergonomics 48(8):1020-1030

Koski L, Iacoboni M, Duebeau M-Ch, Woods RG, Mazziotta JC (2003) Modulation of cortical activity during different imitative behaviors. J Neurophysiol 89:460-471

Lakin JL, Jefferis VE, Cheng CM, Chartrand TL (2003) The chameleon effect as social glue: evidence for the evolutionary significance of nonconscious mimicry. J Nonverbal Behav 27:145-162

Mottet D, Guiard Y, Ferrand Th, Bootsma RJ (2001). Two-handed performance of a rhythmical Fitts task by individuals and dyads. J Exp Psychol Hum Percept Perform 27:1275-1286

Nowak DA, Hermsdorfer J (2003) Sensorimotor memory and grip force control: does grip force anticipate a self-produced weight change when drinking with a straw from a cup? Eur J Neurosci 18:2883-2892

Oztop EW, Wolpert D, Kawato M (2005) Mental state inference using visual control parameters. Cogn Brain Res 22:129-151

Rizzolatti G, Craighero L (2004) The mirror-neuron system. Ann Rev Neurosci 27:169-192

Rosenbaum DA, Weber RJ, Hazelett WM, Hindorff V (1986) The parameter remapping effect in human performance: evidence from tongue twisters and finger fumblers. J Mem Lang 25:710-725

Sebanz N, Bekkering H, Knoblich G (2006). Joint action: bodies and minds moving together. Trends Cogn Sci 10:70-76

Schmidt RC, O'Brien B (1997) Evaluating the dynamics of unintended interpersonal coordination. Ecol Psychol 9:189-206

Shockley K, Santana MV, Fowler CA (2003) Mutual interpersonal postural constraints are involved in cooperative conversation. J Exp Psychol Hum Percept Perform 29:326-332

Van Schie HT, Mars RB, Coles MGH (2004) Modulation of activity in medial frontal and motor cortices during error observation. Nat Neurosci 7:549-554

Wolpert DM, Doya K, Kawato M (2003) A unifying computational framework for motor control and social interaction. Philos Trans R Soc Lond B 358:593-602 